IOS Press

\title{
Does mobilisation of the thoracic spine using mechanical massage affect diaphragmatic excursion in individuals with thoracic hyperkyphosis?
}

\author{
Sung-Hoon Jung, Ui-Jae Hwang, Sun-Hee Ahn, Jun-Hee Kim and Oh-Yun Kwon* \\ Department of Physical Therapy, Kinetic Exercise Based on Movement Analysis Laboratory, College of Health \\ Science, Yonsei University, Wonju, South Korea
}

Received 27 May 2021

Accepted 31 August 2021

\begin{abstract}
.
BACKGROUND: Thoracic mobilisation improves thoracic hyperkyphosis and respiratory function. Diaphragmatic excursion is associated with respiratory function; however, limited studies have assessed the effect of thoracic mobilisation on diaphragmatic excursion.

OBJECTIVE: This study aimed to investigate the effects of thoracic mobilisation on diaphragmatic excursion and respiratory function in individuals with thoracic hyperkyphosis.

METHODS: Participants were recruited through Internet advertising and participated voluntarily. Nineteen healthy participants (age: $33.37 \pm 6.56$ years; height: $170.32 \pm 7.92 \mathrm{~cm}$; weight: $69.77 \pm 14.70 \mathrm{~kg}$ ) with thoracic hyperkyphosis underwent thoracic mobilisation for 8 weeks. Diaphragmatic excursion, thoracic kyphosis, and respiratory function were measured. Thoracic mobilisation was provided using a mechanical massage device.

RESULTS: Thoracic mobilisation for 8 weeks significantly improved diaphragmatic excursion during deep breathing $(p=0.015)$, forced vital capacity $(p<0.01)$, and thoracic hyperkyphosis $(p<0.01)$.

CONCLUSIONS: Thoracic mobilisation can be recommended in respiratory rehabilitation programs to increase diaphragmatic excursion and respiratory function for the management and prevention of respiratory dysfunction in individuals with thoracic hyperkyphosis.
\end{abstract}

Keywords: Diaphragm, thoracic vertebra, massage, kyphosis, respiratory

\section{Introduction}

Thoracic kyphosis is the sagittal plane of the thoracic spine measured using the Cobb angle. When the thoracic kyphosis angle is $>40^{\circ}$, it is defined as thoracic hyperkyphosis [1-4]. Thoracic kyphosis is negatively correlated with thoracic expansion [5]. Restriction of thoracic expansion reduces rib cage excursion, which

${ }^{*}$ Corresponding author: Oh-Yun Kwon, 234 Maeji-ri, HeungeupMyeon, Wonju, Kangwon-Do 26493, South Korea. Tel.: +82 33760 2427; Fax: +82 33760 2496; E-mail: kwonoy@yonsei.ac.kr. in turn reduces respiratory function [5-10]. Therefore, thoracic kyphosis is associated with respiratory function.

Mobilisation has been applied to improve respiratory function in the thoracic spine in the posteroanterior direction [11-16]. Applying posteroanterior mobilisation to the thoracic spine improves the angle of thoracic kyphosis and mobility of thoracic extension, leading to an upright posture $[17,18]$. Jung and Moon reported that thoracic mobilisation over 18 sessions for 6 weeks significantly improved chest expansion. The upright posture has a higher respiratory function than 
the slouched posture, suggesting reduced diaphragm tension and mobility [19,20]. Thus, a slouched posture eventually decreases the ability of the diaphragm, such as that observed in diaphragmatic excursion [20].

Diaphragmatic excursion increases the volume of the thoracic cavity in both the anteroposterior and mediolateral diameters $[21,22]$. As the volume of the thoracic cavity increases, the pressure in the thoracic cavity becomes lower than the atmospheric pressure, and the pressure difference causes inspiration [21]. Therefore, diaphragmatic excursion is correlated with respiratory function [23]. Cardenas et al. reported that there are significant correlations between diaphragmatic excursion and forced vital capacity $(r=0.54)$ and forced expiratory volume in $1 \mathrm{~s}(r=0.53)$. The diaphragm is attached to the internal surfaces of the rib cage and sternum, and diaphragmatic excursion during deep breathing is accompanied by a slight extension of the thoracic spine [22,24]. Individuals with thoracic hyperkyphosis may have reduced thoracic extension during diaphragmatic excursion [25,26].

Since there is a negative correlation between thoracic kyphosis and diaphragmatic excursion, diaphragmatic excursion may improve with improvement in hyperkyphosis [26]. Improvements in hyperkyphosis also increase respiratory function. Therefore, this study aimed to investigate how thoracic mobilisation, which improves hyperkyphosis, affects diaphragmatic excursion and respiratory function in patients with thoracic hyperkyphosis. We hypothesized that the thoracic mobilisation for 8 weeks improves diaphragmatic excursion and respiratory function in patients with thoracic hyperkyphosis.

\section{Methods}

\subsection{Participants}

Participants were recruited through Internet advertising and participated voluntarily from July 2018 to August 2019. Healthy participants aged 18-50 years with thoracic kyphosis angle $>40^{\circ}$ [27], measured using a spinal mouse (Idiag AG, Fehraltorf, Switzerland), were included in the study. However, participants with the following characteristics were excluded: presence of scoliosis, a history of spinal column fracture, spinal tumours and related malignancies, congenital spinal anomalies, cancer, and rheumatoid arthritis [18].

Based on a pilot study, the required sample size was calculated by a priori power analysis using G*Power
3.1 (G*Power Software Inc., Kiel, Germany) using the effect size calculation of diaphragmatic excursion, the $\alpha$ level was set to 0.05 . After conducting a pilot study with 5 participants in this study, a minimum sample size of 18 was required to obtain a power of 0.95 .

A total of 94 participants were screened, and 47 participants who met the exclusion criteria were excluded. Among the participants who met the inclusion criteria, 20 were enrolled according to the calculation of the sample size in this study. During the intervention, 1 participant was excluded for personal reasons. Nineteen subjects (age: $33.37 \pm 6.56$ years; height: $170.32 \pm$ $7.92 \mathrm{~cm}$; weight: $69.77 \pm 14.70 \mathrm{~kg}$; body mass index: $23.83 \pm 3.66 \mathrm{~kg} / \mathrm{m}^{2}$, male/female: $12 / 7$ ) were finally included in this study.

The study was approved by the Yonsei University Mirae Institutional Review Board (1041849-201901BM-019-01), and was registered by the Korea Clinical Research Information Service (KCT0004527; Registered on 7 December 2019, https://cris.nih.go.kr/cris/en/ search/search_result_st01.jsp?seq=15268). All participants provided written informed consent before inclusion.

\subsection{Thoracic mobilisation}

Thoracic mobilisation is externally imposed, with small-amplitude passive motion that is intended to produce gliding at the thoracic spine joint [28] . To apply thoracic mobilisation, a mechanical massage device (Pookjam Factorial Inc., Seoul, South Korea) with mobilisation effect was used [3]. This device was a mat-type mechanical mobilisation/massage device that had two 5-cm-diameter circular rollers located $7.8 \mathrm{~cm}$ apart. The rollers massaged the erector spinae muscles bilaterally while simultaneously mobilising the spine in the direction of extension. For the thoracic spine, the thoracic mode of the device was used with roller massage. This thoracic mode provided mobilisation from the head to the thoracic spine. The rollers moved in upward and downward motion with the same speed of $0.4 \mathrm{~cm} / \mathrm{s}$, and in the thoracic mode, a total of 3 cycles were performed for $25 \mathrm{~min}$. The device was applied at the participant's home and applied for 3 sessions per week (a total of 24 sessions for 8 weeks) (Fig. 1). In order to supervise whether the participant performed all interventions steadily, the supervisor confirmed whether the participant was performing the intervention using a message. All participants faithfully participated in 24 interventions, and there were no reported adverse events. 


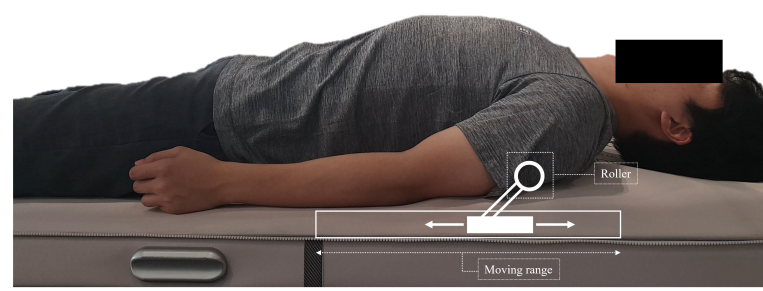

Fig. 1. Application of the mechanical massage device and mimetic diagram of the roller.

\subsection{Outcomes}

\subsubsection{Diaphragmatic excursion}

Diaphragmatic excursions were recorded using a portable ultrasound system (A35; Samsung Medison, Seoul, South Korea) and a sector transducer (3.5 MHz). The experimental method has been reported elsewhere [23] and has a high intra-rater reliability [29]. To evaluate diaphragmatic excursion, the participants were placed in the supine position, and the probe was placed on the abdominal region between the midclavicular and anterior axillary lines. The transducer was directed medially, cephalad, and dorsally so that the ultrasound beam was perpendicular to the posterior third of the right hemidiaphragm. Diaphragmatic excursion was assessed using M-mode imaging between the end of expiration and the end of inspiration during tidal and deep breathing (Fig. 2). Before measurement, participant was instructed to perform deep breathing task and familiarisation was performed 1-2 times. We recorded the average of two consecutive measurements of tidal breathing and the average of four measurements of deep breathing.

\subsubsection{Thoracic kyphosis}

Digital lateral thoracic spine radiographs were obtained using a collimator (Medien International, Co. Ltd., Gyeonggi-do, South Korea) and MC-D computed radiographic systems (Medien International, Co., Ltd., Gyeonggi-do, South Korea) to measure thoracic kyphosis. The measurement of thoracic kyphosis has high inter -and intra-rater reliability $[17,30]$. Each participant stood at a distance of $100 \mathrm{~cm}$ so that the left side of the body faced the X-ray beam, and the beam was centred at $\mathrm{T} 7$ of the thoracic spine [31,32]. All image analyses were performed by one investigator who also performed a repeatability study of the images. The radiographic images were analysed on a personal computer using Image J (National Institutes of Health, Bethesda, MD, USA). For analysis of the radiographic images, the angles of the two lines between the superior end plate of $\mathrm{T} 4$ and the inferior end plate of T12 were analysed [33].

\subsubsection{Respiratory function test}

To investigate changes in respiratory function, we measured the forced vital capacity, forced expiratory volume in $1 \mathrm{~s}$, and peak expiratory flow. These variables were measured using a digital spirometer (SP10; Contec Medical, China). All participants sat on a stool and looked straight. Participants were instructed how to test with a spirometer, and familiarisation was performed 1-2 times. For the test, the participant breathed into the mouthpiece of the spirometer after inhalation to the maximum when the participant's nose was clamped with a nose clip [34]. We recorded the average of two consecutive measurements. The forced vital capacity and forced expiratory volume at one second \%predicted value was determined according to Hankinson's study [35].

\subsection{Statistical analysis}

The Kolmogorov-Smirnov test was used to assess the homogeneity of variance of diaphragmatic excursion $(p>0.05)$. To compare the effects before and after thoracic mobilisation, a paired t-test was used. Intra-rater reliability for measurements of diaphragmatic excursion and respiratory function was calculated using the intraclass correlation coefficient (ICC) $(3,1)$ model. All statistical analyses were performed using the Statistical Package for the Social Sciences version 20.0. (IBM, Armonk, NY), and the level of statistical significance was set at $p<0.05$.

\section{Results}

The results of the paired $t$-test for diaphragmatic excursion and respiratory function are shown in Table 1. Significant differences in diaphragmatic excursion during deep breathing and forced vital capacity were evident. The thoracic kyphosis angle improved significantly after device-assisted intervention combining mobilisation and massage (pre $=43.43^{\circ} \pm 6.76^{\circ}$, post $=$ $39.11^{\circ} \pm 6.74^{\circ}, t=3.416, p<0.01$; Fig. 3). Intra-rater reliability for measurements was statistically significant (diaphragmatic excursion during tidal breathing: ICC $=0.939,95 \%$ confidence interval $[\mathrm{CI}]=0.877-0.974$; diaphragmatic excursion during deep breathing: ICC = $0.961,95 \% \mathrm{CI}=0.899-0.985$; forced vital capacity: ICC $=0.970,95 \% \mathrm{CI}=0.922-0.988$; forced expiratory volume in $1 \mathrm{~s}$ : $\mathrm{ICC}=0.975,95 \% \mathrm{CI}=0.934$ 0.990; peak expiratory flow: $\mathrm{ICC}=0.974,95 \% \mathrm{CI}=$ 0.933-0.990). 

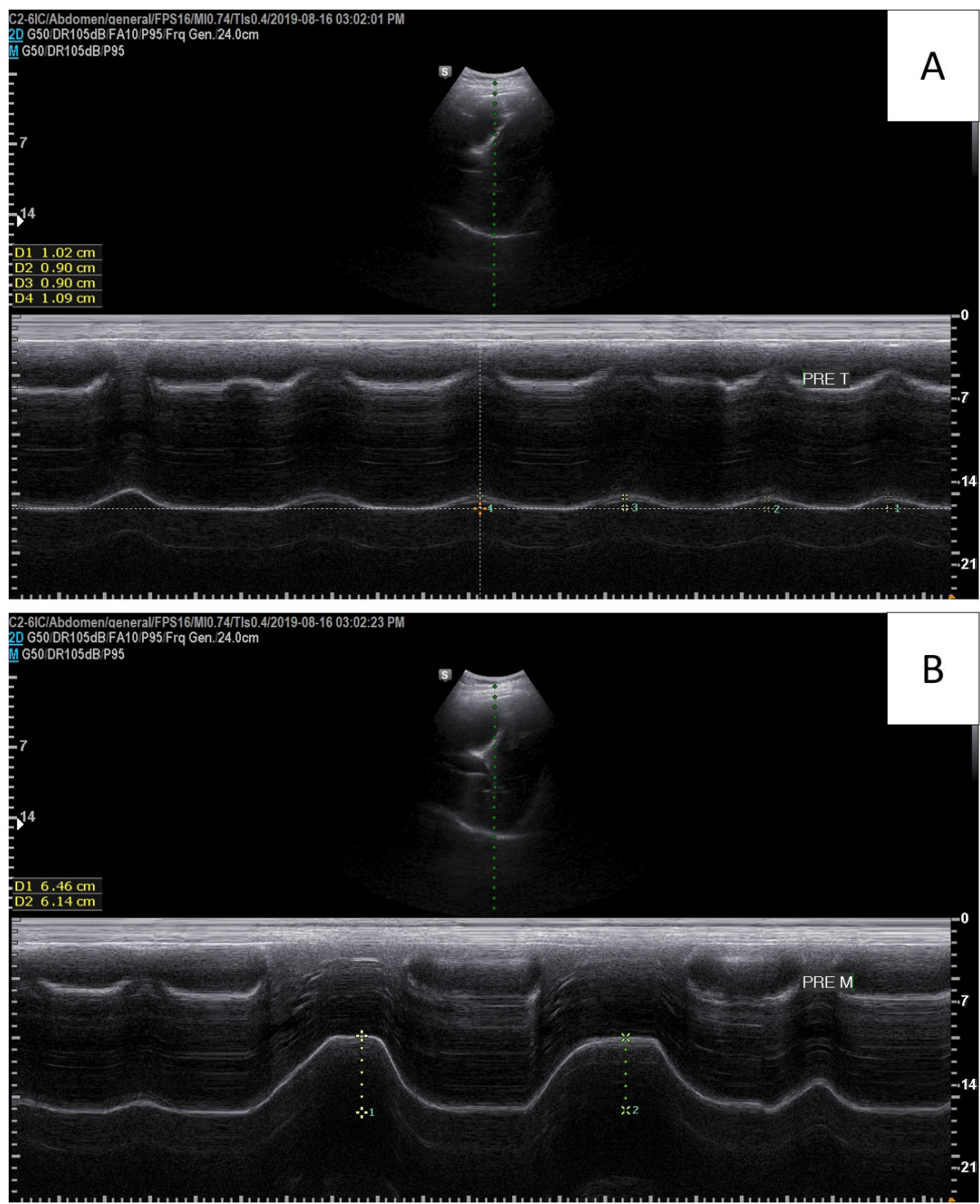

Fig. 2. Diaphragmatic excursion measured by an ultrasound system. (A): Tidal breathing, (B): Deep breathing.

\section{Discussion}

Various studies have previously reported that respiratory function improves after thoracic mobilisation [12-14,36,37]. The purpose of this study was to investigate the improvement in diaphragmatic excursion after thoracic mobilisation for 8 weeks in individuals with thoracic hyperkyphosis. We observed an improvement in diaphragmatic excursion during deep breathing in individuals with hyperkyphosis after mobilisation for 8 weeks $(p=0.015)$. In addition, improvements in forced vital capacity and thoracic hyperkyphosis were observed ( $p<0.01$ ), consistent with the results of previous studies $[13,14,18]$.
Diaphragmatic excursion is related to the lengthtension relationship in the apposition zone of the diaphragm $[25,26]$. If the diaphragm does not have an ideal length-tension relationship, diaphragmatic excursion decreases according to the muscle length-tension relationship [38]. Thoracic hyperkyphosis restricts chest expansion and alters the ideal length-tension relationship of the diaphragm, thus reducing diaphragmatic excursion $[5,12,26,39,40]$. In our study, diaphragmatic excursion during deep breathing improved from $5.53 \mathrm{~mm}$ to $6.26 \mathrm{~mm}$ after 8 weeks of mobilisation in patients with thoracic hyperkyphosis $(p=0.015)$. In addition, mobilisation for 8 weeks improved thoracic hyper- 
Table 1

Diaphragm excursion and respiratory functions before and after thoracic mobilisation

\begin{tabular}{lccccc}
\hline & $\begin{array}{c}\text { Pre-mobilisation } \\
(\text { mean } \pm \text { SD) }\end{array}$ & $\begin{array}{c}\text { Post-mobilisation } \\
(\text { mean } \pm \text { SD) }\end{array}$ & $t$ & Sig. & Effect size \\
\hline Diaphragm excursion during tidal breathing (cm) & $1.62 \pm 0.32$ & $1.71 \pm 0.35$ & -1.027 & 0.318 & 0.236 \\
Diaphragm excursion during deep breathing (cm) & $5.53 \pm 1.39$ & $6.26 \pm 0.83$ & -2.699 & $0.015^{*}$ & 0.619 \\
Forced vital capacity (L) & $3.87 \pm 0.81$ & $4.00 \pm 0.81$ & -4.239 & $0.000^{*}$ & 0.972 \\
Forced vital capacity (\%pred) & $82.98 \pm 10.50$ & $85.75 \pm 9.95$ & -3.754 & $0.001^{*}$ & 0.861 \\
Forced expiratory volume in one second (L) & $3.42 \pm 0.68$ & $3.42 \pm 0.72$ & 0.163 & 0.873 & 0.037 \\
Forced expiratory volume in one second (\%pred) & $89.97 \pm 12.82$ & $89.81 \pm 14.97$ & 0.154 & 0.880 & 0.035 \\
Peak expiratory flow (L/s) & $7.08 \pm 1.98$ & $7.42 \pm 2.30$ & -1.600 & 0.127 & 0.367 \\
Tiffeneau-Pinelli index ${ }^{\mathrm{a}}(\%)$ & $0.89 \pm 0.05$ & $0.86 \pm 0.07$ & 1.858 & 0.080 & 0.426 \\
\hline
\end{tabular}

$\mathrm{SD}=$ standard deviation, ${ }^{*} p<0.05,{ }^{a}$ Forced vital capacity/Forced expiratory volume in one second (FEV1\%).

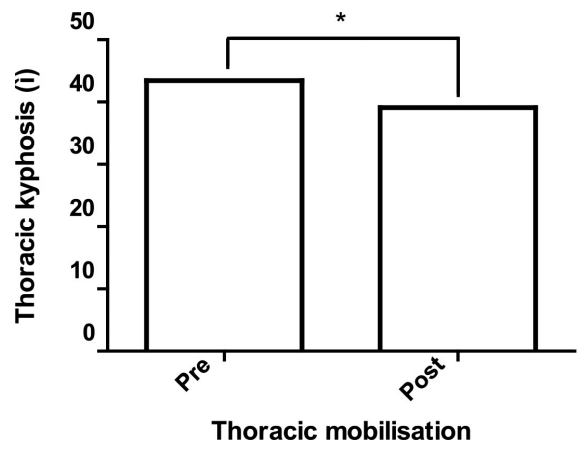

Fig. 3. Thoracic kyphosis before and after thoracic mobilisation.

kyphosis $(p<0.01)$, consistent with the results of previous studies $[18,41]$. This improvement in thoracic hyperkyphosis may have helped optimise the lengthtension relationship of the diaphragm and create a mechanical advantage for diaphragmatic excursion during deep breathing.

Babina et al. [13] reported that forced vital capacity improved more when thoracic mobilisation and breathing exercises were applied together than when only breathing exercises were applied. In our study, forced vital capacity improved after 8 weeks of mobilisation in patients with thoracic hyperkyphosis $(p<0.01)$. Although no variables were measured directly in this study, there are four possible reasons why thoracic mobilisation improved forced vital capacity. First, diaphragmatic excursion improved after thoracic mobilisation. Improving diaphragmatic excursion increases inspiratory muscle strength, which further improves forced vital capacity [23]. Second, chest wall expansion improves after thoracic mobilisation [12]. Improvement in chest wall expansion provides the lung with relatively more space to expand, thus causing an overall improvement in forced vital capacity [42]. Third, the respiratory muscles can be stimulated by thoracic mobilisation. In general, when the muscle contracts, the central stimulus comes from the motor cortex to the spinal level [43].
If the activation of the inter-neuronal pool at the spinal level is hyperexcitable owing to stimulation such as mobilisation, the activation adds to the already present central stimulus. Therefore, stimulation reaching the motor nerve fibres is greater than the original central stimulation [13]. Fourth, back massage improves respiratory rate [44]. Back massage can relax not only the erector spinae muscle, but also the levatore costarum muscles. Therefore, the intervention combining mobilisation and massage would have an effect on respiratory function by improving the movement of the costospinal joint.

Diaphragmatic excursion during deep breathing has a significant correlation with respiratory function, but diaphragmatic excursion during tidal breathing has no significant correlation [23]. In a study by Wang [40], diaphragm excursion during deep breathing was significantly correlated with chest wall expansion; however, diaphragm excursion during tidal breathing had no significant correlation. In our study, diaphragm excursion increased significantly during deep breathing after thoracic mobilisation, but there was no significant difference during tidal breathing, possibly because of the movement of the slight extension of the thoracic spine combined with movement of the ribs during deep breathing. Therefore, it is clinically meaningful to measure the change in diaphragmatic excursion during deep breathing rather than during tidal breathing when investigating the effects of interventions.

\subsection{Limitation and future scope}

This study has several limitations. First, follow-up data were not measured. Therefore, it is difficult to determine the extent to which thoracic mobilisation is maintained after 8 weeks. Second, the control group was not included in this study. Therefore, it is difficult to clearly identify the cause of improved respiration after thoracic mobilisation. In future studies, a control 
group will be needed to investigate the causes of improved respiration after thoracic mobilisation. Third, our study included subjects aged 20-50 years, and the age of the final participants was $33.37 \pm 6.56$ years. Since we did not include patients aged $>50$ years, our findings cannot be generalised to the elderly population. Fourth, the angle of thoracic kyphosis in the study participants was $43.43^{\circ} \pm 6.76^{\circ}$. Because we did not include subjects with severe hyperkyphosis (angle $>$ $50^{\circ}$ ), our findings cannot be generalised to more severe types of thoracic hyperkyphosis. Lastly, only the Cobb angle was measured as a variable in which mobilisation affects the spinal joint. In future studies, various variables such as electromyography, magnetic resonance imaging, and range of motion measurement will also be needed to determine changes in muscle properties and range of motion after thoracic mobilisation.

\section{Conclusions}

We found that a device-assisted intervention combining mobilisation and massage for 8 weeks increased diaphragmatic excursion during deep breathing, forced vital capacity, and improved thoracic hyperkyphosis. Thoracic mobilisation can be recommended in respiratory rehabilitation programs to increase diaphragmatic excursion and respiratory function for the management and prevention of respiratory dysfunction in individuals with thoracic hyperkyphosis.

\section{Acknowledgments}

The authors would like to thank the assistant researcher at KEMA station for the helpful assistance in the preparation of this study.

\section{Conflict of interest}

The authors declare that they have no potential conflicts of interest with respect to the research, authorship, or publication of this article. The results are presented clearly, honestly, and without fabrication, falsification, or inappropriate data manipulation.

\section{Funding}

This work was supported by the Yonsei University Research Fund (2019-51-0321 and 2020-51-0013).

\section{References}

[1] Fon GJ, Pitt MJ, Thies AC. Thoracic kyphosis: Range in normal subjects. Am J Roentgenol. 1980; 134(5): 979-983.

[2] Vaughn DW, Brown EW. The influence of an in-home based therapeutic exercise program on thoracic kyphosis angles. J Back Musculoskelet Rehabil. 2007; 20(4): 155-165.

[3] Jung S, Hwang U, Ahn S, Kim J, Kwon O. Effects of Manual Therapy and Mechanical Massage on Spinal Alignment, Extension Range of Motion, Back Extensor Electromyographic Activity, and Thoracic Extension Strength in Individuals with Thoracic Hyperkyphosis: A Randomized Controlled Trial. Evidence-Based Complement Altern Med. 2020; 2020: 6526935.

[4] Jung S, Hwang U, Kim J, Ha S, Kwon O. Correlation among Non-radiological Measurements for Thoracic Kyphosis. J Musculoskelet Sci Technol. 2020; 4(1): 1-5.

[5] Culham EG, Jimenez HAI, King CE. Thoracic kyphosis, rib mobility, and lung volumes in normal women and women with osteoporosis. Spine. 1994; 19(11): 1250-5.

[6] Teramoto S, Suzuki M, Matsuse T, Ohga E, Katayama H, Nagase $\mathrm{T}$, et al. Influence of Kyphosis on the age-related decline in pulmonary function. Japanese J Geriatr. 1998; 35(1): 23-7.

[7] Lee SJ, Chang JY, Ryu YJ, Lee JH, Chang JH, Shim SS, et al. Clinical Features and Outcomes of Respiratory Complications in Patients with Thoracic Hyperkyphosis. Lung. 2015; 193(6): 1009-15.

[8] Harrison RA, Siminoski K, Vethanayagam D, Majumdar SR. Osteoporosis-related kyphosis and impairments in pulmonary function: A systematic review. Journal of Bone and Mineral Research. 2007; 22(3): 447-57.

[9] Taslimipour S, Rojhani-Shirazi Z, Hemmati L, Rezaei I. Effects of a virtual reality dance training program on kyphosis angle and respiratory parameters in young women with postural hyperkyphosis: a randomized controlled clinical trial. J Sport Rehabil. 2020; 30(2): 293-9.

[10] Kazemi K, Rahmani N, Rahimi F, Ravanbakhsh M. The association between spinal column deformity and breathing function: A systematic review. J Bodyw Mov Ther. 2020; 24(3): 172-80.

[11] Hwangbo PN, Hwangbo G, Park J, Lee S. The effect of thoracic joint mobilization and self-stretching exercise on pulmonary functions of patients with chronic neck pain. J Phys Ther Sci. 2014; 26(11): 1783-6.

[12] Jung JH, Moon DC. The effect of thoracic region selfmobilization on chest expansion and pulmonary function. $\mathbf{J}$ Phys Ther Sci. 2015; 27(9): 2779-81.

[13] Babina R, Mohanty PP, Pattnaik M. Effect of thoracic mobilization on respiratory parameters in chronic non-specific low back pain: A randomized controlled trial. J Back Musculoskelet Rehabil. 2016; 29(3): 587-95.

[14] Jang SH, Bang HS. Effect of thoracic and cervical joint mobilization on pulmonary function in stroke patients. J Phys Ther Sci. 2016; 28(1): 257-60.

[15] Wang JS. Effect of thoracic cage mobilization on respiratory function, spinal curve and spinal movement in patients with restrictive lung disease. J Int Acad Phys Ther Res. 2016; 7(2): 1006-10.

[16] Wang JS. The Effect of Thoracic Cage Mobilization and Breathing Exercise of Respiratory Function, Spinal Curve and Spinal Mobility in Elderly with Restrictive Lung Disease. J Int Acad Phys Ther Res. 2018; 9(1): 1393-7. 
[17] Briggs AM, Van Dieën JH, Wrigley TV, Greig AM, Phillips B, Lo SK, et al. Thoracic kyphosis affects spinal loads and trunk muscle force. Phys Ther. 2007; 87(5): 595-607.

[18] Kamali F, Shirazi SA, Ebrahimi S, Mirshamsi M, Ghanbari A. Comparison of manual therapy and exercise therapy for postural hyperkyphosis: A randomized clinical trial. Physiother Theory Pract. 2016; 32(2): 92-7.

[19] Landers M, Barker G, Wallentine S, McWhorter JW, Peel C. A comparison of tidal volume, breathing frequency, and minute ventilation between two sitting postures in healthy adults. Physiother Theory Pract. 2003; 19(2): 109-119.

[20] Albarrati A, Zafar H, Alghadir AH, Anwer S. Effect of Upright and Slouched Sitting Postures on the Respiratory Muscle Strength in Healthy Young Males. Biomed Res Int. 2018; 2018: 3058970.

[21] Fox SI. Fundamentals of Human Physiology. McGraw-Hill Education. 2016

[22] Neumann DA. Kinesiology of the musculoskeletal system: foundations for rehabilitation. Mosby Elsevier, St. Louis MO. 2010

[23] Cardenas LZ, Santana PV, Caruso P, Ribeiro de Carvalho CR, Pereira de Albuquerque AL. Diaphragmatic Ultrasound Correlates with Inspiratory Muscle Strength and Pulmonary Function in Healthy Subjects. Ultrasound Med Biol. 2018; 44(4): 786-793.

[24] Muscolino JE. Know the body: muscle, bone, and palpation essentials-e-book. Elsevier Health Sciences; 2013.

[25] Cassart M, Pettiaux N, Gevenois PA, Paiva M, Estenne M. Effect of chronic hyperinflation on diaphragm length and surface area. Am J Respir Crit Care Med. 1997; 156(2 Pt 1): 504-8.

[26] Aparecida Gonçalves M, Estima Leal B, Da Cunha Viegas G, Nunes Lúcio M, Zarpellon Mazo G, Paulin E. The relation between diaphragmatic mobility and spinal curvatures in patients with chronic obstructive pulmonary disease. Fisioter e Pesqui. 2017; 24: 245-252.

[27] Katzman WB, Wanek L, Shepherd JA, Sellmeyer DE. Agerelated hyperkyphosis: Its causes, consequences, and management. Journal of Orthopaedic and Sports Physical Therapy. 2010; 40(6): 352-360

[28] Edmond SL. Joint Mobilization/Manipulation-E-Book: Extremity and Spinal Techniques. Elsevier Health Sciences, 2016.

[29] Nassiri K, Abedi M, Baghban AA, Meymeh MH, Manshadi FD. Comparison of Diaphragm Thickness and Excursion between Patients with Pelvic Girdle Pain and Healthy Individuals. J Clin Physiother Res. 2020; 5(2): e12-e12.

[30] Porto AB, Okazaki VHA. Thoracic kyphosis and lumbar lordosis assessment by radiography and photogrammetry: a review of normative values and reliability. J Manipulative Physiol Ther. 2018; 41(8): 712-23.

[31] Edmondston SJ, Christensen MM, Keller S, Steigen LB, Barclay L. Functional radiographic analysis of thoracic spine extension motion in asymptomatic men. J Manipulative Physiol Ther. 2012; 35(3): 203-208.

[32] Gallagher KM, Sehl M, Callaghan JP. A radiographic assessment of lumbar spine posture in four different upright standing positions. Clin Biomech. 2016; 37: 131-136.

[33] Katzman WB, Parimi N, Gladin A, Poltavskiy EA, Schafer AL, Long RK, et al. Sex differences in response to targeted kyphosis specific exercise and posture training in communitydwelling older adults: a randomized controlled trial. BMC Musculoskelet Disord. 2017; 18(1): 1-11.

[34] Kang KW, Jung SI, Lee DY, Kim K, Lee NK. Effect of sitting posture on respiratory function while using a smartphone. J Phys Ther Sci. 2016; 28(5): 1496-1498.

[35] Hankinson JL, Odencrantz JR, Fedan KB. Spirometric reference values from a sample of the general US population. Am J Respir Crit Care Med. 1999; 159(1): 179-87.

[36] Yilmaz Yelvar GD, çirak Y, Parlak Demir Y, Dalkilinç M, Bozkurt B. Immediate effect of manual therapy on respiratory functions and inspiratory muscle strength in patients with COPD. Int J COPD. 2016; 11: 1353-7.

[37] Cruz-Montecinos C, Godoy-Olave D, Contreras-Briceño FA, Gutiérrez P, Torres-Castro R, Miret-Venegas L, et al. The immediate effect of soft tissue manual therapy intervention on lung function in severe chronic obstructive pulmonary disease. Int J COPD. 2017; 12: 691-696.

[38] Kisner C, Colby LA. Therapeutic Exercise Foundations and Techniques, Sixth Edition, FA. Davis Company, Am. 2012.

[39] Frownfelter Donna L, Elizabeth D, eds., Principles and Practice of Cardiopulmonary Physical Therapy. Mosby Incorporated, 1996.

[40] Wang HK, Lu TW, Liing RJ, Shih TTF, Chen SC, Lin KH. Relationship Between Chest Wall Motion and Diaphragmatic Excursion in Healthy Adults in Supine Position. J Formos Med Assoc. 2009; 108(7): 577-86.

[41] Bautmans I, Van Arken J, Van Mackelenberg M, Mets T. Rehabilitation using manual mobilization for thoracic kyphosis in elderly postmenopausal patients with osteoporosis. J Rehabil Med. 2010; 42(2): 129-35.

[42] Kakizaki F, Shibuya M, Yamazaki T, Yamada M, Suzuki H, Homma I. Preliminary report on the effects of respiratory muscle stretch gymnastics on chest wall mobility in patients with chronic obstructive pulmonary disease. Respir Care. 1999; 19(6): 390-391.

[43] Pollard H, Ward G. Strength change of quadriceps femoris following a single manipulation of the $\mathrm{L} 3 / 4$ vertebral motion segment: A preliminary investigation. JNMS - J Neuromusculoskel Syst. 1996; 4(4): 137-144.

[44] Chen W-L, Liu G-J, Yeh S-H, Chiang M-C, Fu M-Y, Hsieh Y-K. Effect of back massage intervention on anxiety, comfort, and physiologic responses in patients with congestive heart failure. J Altern Complement Med. 2013; 19(5): 464-70. 